\title{
Characterization of Ti Ringspot-Associated Virus, a Novel Emaravirus Associated with an Emerging Ringspot Disease of Cordyline fruticosa
}

\author{
Alejandro Olmedo-Velarde, Adam C. Park, Jari Sugano, Janice Y. Uchida, Michael Kawate, Wayne B. Borth, \\ John S. Hu, and Michael J. Melzer ${ }^{\dagger}$ \\ Department of Plant and Environmental Protection Sciences, University of Hawaii, Honolulu, HI 96822
}

\begin{abstract}
Ti ringspot is an emerging foliar disease of the ti plant (Cordyline fruticosa) in Hawaii that is quickly spreading throughout the islands. Symptoms include small chlorotic ringspots on leaves that often coalesce to form larger lesions. Although several virus species have been discovered in symptomatic plants, none have been associated with these symptoms. Here, we report and characterize a novel virus closely associated with ti ringspot symptoms in Hawaii. The presence of double membrane bodies approximately $85 \mathrm{~nm}$ in diameter in symptomatic cells and sequence analyses of five genomic RNA segments obtained by high-throughput sequencing indicate that this virus is most closely related to members of the plant virus genus Emaravirus. Phylogenetic

and sequence homology analyses place this virus on a distinct clade within the Emaravirus genus along with High Plains wheat mosaic emaravirus, blue palo verde broom virus, and Raspberry leaf blotch emaravirus. Sequence identity values with taxonomically relevant proteins indicate that this represents a new virus species, which we are tentatively naming ti ringspot-associated virus (TiRSaV). TiRSaVspecific reverse transcription PCR assays detected the virus in several experimental herbaceous host species following mechanical inoculation. TiRSaV was also detected in eriophyid mites collected from symptomatic ti plants, which may represent a putative arthropod vector of the virus.
\end{abstract}

The genus Emaravirus (family Fimoviridae, order Bunyavirales) is composed of plant viruses with negative-sense, multipartite ssRNA genomes. Members of this genus possess four core segments that individually encode for RNA-dependent RNA polymerases (RdRps), glycoprotein precursors (GPs), nucleocapsid proteins (NCs), and movement proteins (MPs) (Elbeaino et al. 2018; Mielke-Ehret and Mühlbach 2012; Mühlbach and Mielke-Ehret 2012). Some emaraviruses appear to possess up to four additional segments encoding proteins with unknown function ( $\mathrm{Lu}$ et al. 2015; Tatineni et al. 2014). Double membrane bodies (DMBs) between 80 and $200 \mathrm{~nm}$ in diameter are associated with infection and may represent the Emaravirus virion (Elbeaino et al. 2018; Mielke-Ehret and Mühlbach 2012; Mühlbach and Mielke-Ehret 2012). The Emaravirus genus was established in 2011 and is currently composed of nine species: the type species European mountain ash ringspot-associated emaravirus (EMARaV), Actinidia chlorotic ringspot-associated emaravirus (AcCRaV), Fig mosaic emaravirus (FMV), High Plains wheat mosaic emaravirus (HPWMoV), Pigeonpea sterility mosaic emaravirus 1 (PPSMV 1), Pigeonpea sterility mosaic emaravirus 2 (PPSMV 2), Raspberry leaf blotch emaravirus (RLBV), Redbud yellow ringspot-associated emaravirus (RYRSaV), and Rose rosette emaravirus (RRV). Blackberry leaf mottle-associated virus (BLMaV) (Hassan et al. 2017), Woolly burdock yellow vein virus (Bi et al. 2012), and blue palo verde broom virus (PVBV) (Ilyas et al. 2018) are putative emaraviruses. Eriophyid mites (Acari: Eriophyidae) have been shown to vector the emaraviruses and putative emaraviruses (Di Bello et al. 2016;

${ }^{\dagger}$ Corresponding author: M. J. Melzer; melzer@hawaii.edu

Funding: This work was supported by the U.S. Department of Agriculture National Institute of Food and Agriculture (grant HAW-09030H).

*The $\boldsymbol{e}$-Xtra logo stands for "electronic extra" and indicates that four supplementary figures and one supplementary table are published online.

The author(s) declare no conflict of interest.

Accepted for publication 8 April 2019.

(c) 2019 The American Phytopathological Society
Hassan et al. 2017; McGavin et al. 2012; Mielke-Ehret and Mühlbach 2012).

$\mathrm{Ti}$ (Cordyline fruticosa L.) is a common ornamental and landscaping plant in Hawaii that also has great importance in the Hawaiian culture. Its foliage is used in both the cut flower and food industries. In the latter, ti leaves are used to wrap food prior to cooking but they are not eaten. In historical times, the large corms of ti plants were steamed and eaten or fermented and often further distilled into alcoholic beverages. Given these diverse uses, a small commercial ti industry persists in Hawaii. Ti ringspot is an emerging disease of ti reported by commercial ti growers on the island of Oahu in 2009 (Melzer et al. 2011). The disease has since spread rapidly through much of Oahu and can now be found on the islands of Maui and Hawaii. The hallmark of ti ringspot is the presence of chlorotic rings on the leaf that are typically 0.5 to $1 \mathrm{~cm}$ in diameter (Fig. 1). Occasionally these rings become necrotic, especially on older leaves, or coalesce into larger, amorphous lesions. Unlike some fungal diseases of ti, such as Cercospora leaf spot, the center of the ring does not become necrotic. New leaves emerge asymptomatic and can remain asymptomatic for weeks before developing ringspots, suggesting that the causal agent is either not systemic or symptoms are only expressed in the mature foliage. Green-leaved varieties are most commonly afflicted by ti ringspot, although red-leaved ornamental varieties with ringspot symptoms have been observed (Fig. 1). In red-leaved varieties, the margin of the ringspot is often a light green color, likely owing to a reduction in levels of both chlorophyll and anthocyanin. An unidentified species of eriophyid mite is consistently observed on both symptomatic and asymptomatic ti plants.

Cordyline virus 1 (CoV-1; genus Velarivirus, family Closteroviridae), CoV-2, CoV-3, and CoV-4 were previously detected in ti plants with ti ringspot disease (Melzer et al. 2011, 2013a, b). It was determined, however, that these four viruses are not involved in the etiology of ti ringspot (Melzer et al. 2013a). Chlorotic and necrotic ringspots associated with infection by Orchid fleck dichorhavirus (OFV; family Rhabdoviridae) have recently been reported on C. fruticosa $(=C$. terminalis) in Australia (Dietzgen et al. 2018), although these symptoms appear distinct from ti ringspot disease. Tomato spotted wilt tospovirus (TSWV; family Tospoviridae) has also been reported to infect ti plants, although symptoms of infection have not been described (Cho et al. 1986). With the causal agent of ti ringspot unknown, the purpose of this study was to identify a pathogen 
associated with the disease. Here, we identify and characterize a novel virus with properties similar to members of the genus Emaravirus. The virus is associated with ti ringspot, and we have tentatively designated it as ti ringspot-associated virus (TiRSaV).

\section{Materials and Methods}

Tissue collection and virus source. The primary sources of common green ti plants with symptoms of ti ringspot were a commercial ti farm located in Waihee Valley and the University of Hawaii at Manoa (UHM) campus, both of which are on the island of Oahu. These samples were used for transmission electron microscopy, virus detection, and genomic sequencing. Additional symptomatic and asymptomatic leaf tissue of common green ti plants was collected from the islands of Kauai, Oahu, Maui, and Hawaii for virus detection assays. Leaf lamina from symptomatic, asymptomatic furled, and asymptomatic unfurled leaves as well as petiole, stem, and root tissues were collected from two common green ti plants with ti ringspot growing at the UHM campus to determine the distribution of the putative causal agent in planta.

Transmission electron microscopy. Common green ti leaf pieces approximately $1 \times 2 \mathrm{~mm}$ excised from the margin of ringspot lesions as well as asymptomatic controls were fixed for $2 \mathrm{~h}$ in $0.1 \mathrm{M}$ sodium cacodylate ( $\mathrm{pH} 7.4$ ) containing $2 \%$ glutaraldehyde, $2 \%$ paraformaldehyde, and $2 \mathrm{mM}$ of $\mathrm{CaCl}_{2}$ followed by two 20 -minute washes in $0.1 \mathrm{M}$ of sodium cacodylate solution. Leaf pieces were postfixed in $0.1 \mathrm{M}$ of sodium cacodylate $(\mathrm{pH} 7.4)$ containing $1 \%$ osmium tetroxide for $1.5 \mathrm{~h}$. The leaf pieces were then dehydrated in a graded ethanol series followed by three 10-min exposures to propylene oxide. The leaf pieces were embedded in LX-112 epoxy resin (Ladd Research, Williston, VT) following the manufacturer's instructions. Ultrathin sections were stained with uranyl acetate and lead citrate and viewed with a HT7700 Transmission Electron Microscope (Hitachi High Technologies America Inc., Dallas, TX) at the University of Hawaii Biological Electron Microscope Facility.

Assays for TSWV and OFV. To determine whether TSWV was present in plants with ti ringspot symptoms, a lateral flow assay (LFA) and an enzyme-linked immunosorbent assay (ELISA) specific for TSWV were performed following the manufacturer's instructions (Agdia Inc., Elkhart, IN). Tomato (Solanum lycopersicum) and basil (Ocimum basilicum) tissue infected with TSWV served as positive controls for these assays. To determine whether OFV was present in ti plants with ti ringspot symptoms, reverse transcription (RT) PCR using primers targeting a citrus strain of OFV was performed (Roy et al. 2014). Since no positive control was available for this

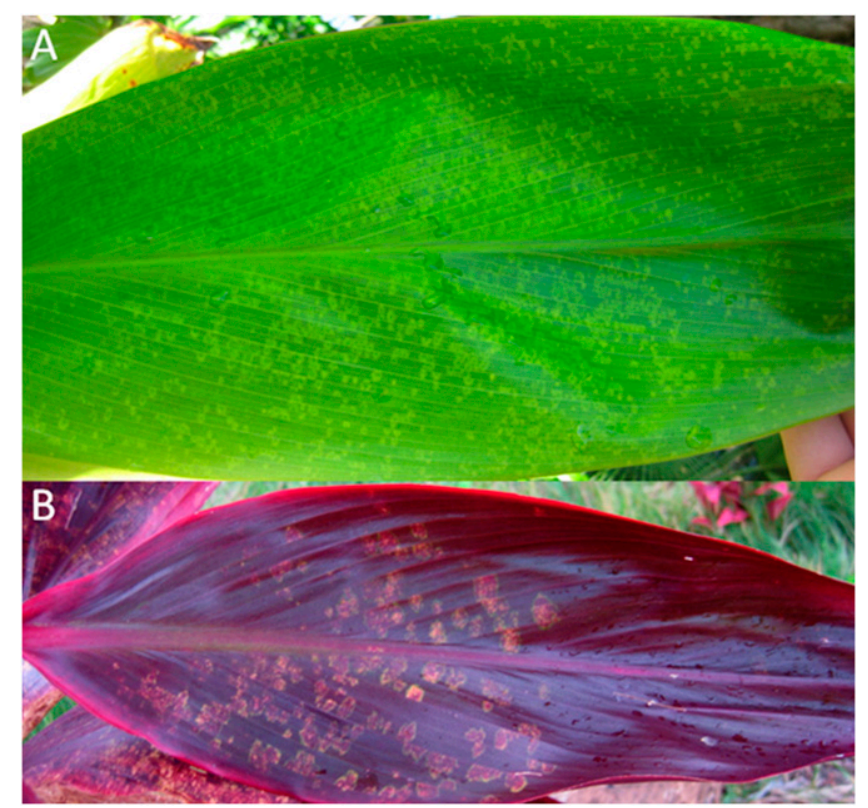

Fig. 1. Ringspot symptoms on the leaves of common $\mathbf{A}$, green and $\mathbf{B}$, red varieties of ti (Cordyline fruticosa). assay, the quality of the RNA extracted from symptomatic tissue was instead evaluated by RT-PCR using internal control primers targeting ti rubisco large subunit $(r b c L)$ mRNA as described (Melzer et al. 2013a).

Emaravirus and TiRSaV detection. Total RNA was isolated from ti tissues using a NucleoSpin RNA Plus kit (Macherey-Nagel, Düren, Germany) and reverse transcribed into cDNA using random primers and the M-MLV reverse transcription kit (Promega, Madison, WI). The cDNA was used as template in a conventional RTPCR assay using Emaravirus-specific degenerate primers (Motif A-sense/Motif C-antisense) targeting RdRp gene motifs described by Elbeaino et al. (2013). Fig tissue infected with FMV was used as a positive control for this assay. Amplification products were cloned using pGEM-T Easy (Promega) and sequenced at the University of Hawaii Advanced Studies in Genomics, Proteomics, and Bioinformatics Laboratory. Primers targeting the $r b c L$ of $C$. fruticosa were used to ensure the quality of template used in the RT-PCR assay (Melzer et al. 2013a).

For specific detection of TiRSaV and association of this virus with the observed symptoms, a conventional RT-PCR detection assay was developed. Primers TiRSaV forward $(\mathrm{F})$ and TiRSaV reverse $(\mathrm{R})$ targeted a portion of the RdRp of TiRSaV, and RBC L3 and RBC R3 targeted a portion of the $r b c L$ of $C$. fruticos $a$ and served as an internal positive control for the detection assay (Supplementary Table S1). These primer pairs were used in both singleplex and multiplex formats to evaluate leaf tissue from 51 symptomatic plants and 23 asymptomatic plants growing at 14 locations on the islands of Kauai, Oahu, Maui, and Hawaii. The PCR cycling conditions were initial denaturation for $5 \mathrm{~min}$ at $95^{\circ} \mathrm{C}$, followed by 40 cycles of $95^{\circ} \mathrm{C}$ for $30 \mathrm{~s}, 55^{\circ} \mathrm{C}$ for $30 \mathrm{~s}$, and $72^{\circ} \mathrm{C}$ for $30 \mathrm{~s}$. A 7 -min final extension was conducted at $72^{\circ} \mathrm{C}$. PCR products were resolved on a $2 \%$ (wt/ vol) agarose gel and visualized with ethidium bromide over ultraviolet light.

Double-stranded RNA isolation and sequencing. Doublestranded RNA (dsRNA) was isolated from symptomatic tissues from ti plants located at the UHM campus using either CF-11 (Whatman, Maidstone, UK) or Sigmacell Type 101 (Sigma, St. Lois, MO) cellulose chromatography following the procedure of Morris and Dodds (1979). These dsRNAs were used as template for creating a degenerate oligo-primed PCR library (Melzer et al. 2011). This library underwent high-throughput sequencing (HTS) using an Illumina HiSeq $2 \times$ $100 \mathrm{bp}$ (V2) platform at SeqMatic LLC (Fremont, CA), which yielded partial segment sequences. A second approach was adopted to obtain additional RNA segments. Briefly, 200 to $300 \mathrm{ng}$ of dsRNA and $1 \mathrm{pmol} /$ liter of primer "Emara terminus," which includes the 13 conserved bases complementary to the conserved $5^{\prime}$ and $3^{\prime}$ terminal sequences in the genomic RNAs of all emaraviruses, were denatured at $95^{\circ} \mathrm{C}$ for $5 \mathrm{~min}$, quickly chilled on ice for $2 \mathrm{~min}$, and reverse transcribed with the M-MLV reverse transcription kit (Promega) at $42^{\circ} \mathrm{C}$ for $1 \mathrm{~h}$. The resulting cDNA was subjected to conventional PCR with primer "Emara terminus" using Phusion High-Fidelity DNA Polymerase (New England Biolabs, Ipswich, MA). The final volume of the reaction was $50 \mu \mathrm{l}$ with cycling parameters consisting of an initial denaturation at $98^{\circ} \mathrm{C}$ for $30 \mathrm{~s}$, followed by 40 cycles of denaturation at $98^{\circ} \mathrm{C}$ for $10 \mathrm{~s}$, annealing at $45^{\circ} \mathrm{C}$ for $20 \mathrm{~s}$, and extension at $72^{\circ} \mathrm{C}$ for $2 \mathrm{~min}$. A final extension was performed for $3 \mathrm{~min}$ at $72^{\circ} \mathrm{C}$. The size of the PCR products was checked using agarose gel electrophoresis, and the PCR products were cleaned and concentrated using Amicon ultra filter YM-30 (Millipore, Darmstadt, Germany) and underwent HTS using an Illumina MiSeq $2 \times 300$ bp (V2) platform at the University of Hawaii Advanced Studies in Genomics, Proteomics, and Bioinformatics Laboratory.

Genome assembly. The single-end and individual paired-end read files from HTS were filtered using Trimmomatic 0.35.3 (Bolger et al. 2014), and reads with phred quality scores lower than 20 were discarded. A quality control check was run with FastQC 0.69 (Babraham Bioinformatics) for the single-end read file and individual paired-end read files, and MultiQC 1.0 (Ewels et al. 2016) was used to merge the quality control files from the individual paired-end reads files. The reads were de novo assembled using Trinity 2.2.0 with 
default parameters (Grabherr et al. 2011) and Velvet 1.2.10 (Zerbino and Birney 2008) with default parameters and kmer sizes ranging from 35 to 65, respectively. Assembled contigs were sorted by length, and duplicates and those with lengths shorter than 100 nucleotides (nt) were discarded using Geneious version 10.1.3 (Kearse et al. 2012). Residual contigs were searched against the nonredundant viral protein database available in GenBank using BlastX (Altschul et al. 1997). Contigs showing resemblance to emaraviruses were identified and saved for further analysis. Using Bowtie 2 (Langmead et al. 2009) and Geneious mappers plug-ins implemented in Geneious version 10.1.3, the $5^{\prime}$ and $3^{\prime}$ ends of partial segments were extended and sequence corrected in some of the contigs, and the genome coverage and read counts were obtained. For bridging sequence gaps, contig-specific primers were used in RT-PCR assays employing dsRNA as template. Amplification products were cloned and sequenced as above. To determine whether contigs putatively representing TiRSaV genomic RNA segments all belonged to the same virus, RT-PCR assays with specific primers to each segment were conducted on seven symptomatic and seven asymptomatic ti plants collected on Oahu. The termini of the viral RNAs 1 to 5 were verified by rapid amplification of cDNA ends (RACE). For both ends, dsRNA was poly(A) tailed using the Escherichia coli poly(A) polymerase (New England Biolabs, Hitchin, UK). Polyadenylated dsRNAs were reverse transcribed using the primer poly(dT) tail RACE and amplified with primers designed to anneal within 450 nt of the terminus. Amplified products were cloned and sequenced as above. All of the bioinformatic tools used, except those implemented in Geneious version 10.1.3, were accessed through the Galaxy platform (Afgan et al. 2016).

Sequence and phylogenetic analyses. The NCBI ORFfinder program (https://www.ncbi.nlm.nih.gov/orffinder/) was used to search for putative open reading frames (ORFs), and the proteins they encoded, in the TiRSaV RNAs identified by HTS. Global pairwise sequence comparisons (amino acid) of the RdRp, GP, NC, MP, and $\mathrm{p} 5$ sequences with orthologs from other emaraviruses were performed using LALIGN (Huang and Miller 1991). The RdRp, GP, $\mathrm{NC}$, and MP amino acid sequences were aligned with their respective orthologs using the MEGA 7.0.25 (Kumar et al. 2016) ClustalW algorithm (Thompson et al. 1994). The best model of protein evolution for each alignment, partial deletion, and moderate branch swap filter were used to generate a maximum likelihood tree with supporting bootstrap values following 1,000 repetitions. Bayesian phylogeny was inferred using BEAST version 2.4.6 (Bouckaert et al. 2014) and the best evolution model with three Markov chain Monte Carlo runs of 100,000,000 generations sampling every 10,000 trees. The process was performed at the CIPRES Science Gateway (http:// www.phylo.org). The three runs were combined using LogCombiner in BEAST, discarding $10 \%$ of the sample trees as burn-in. Effective sample sizes were calculated using Tracer version 1.6 and were $>200$ for all of the parameters. The maximum clade credibility tree and posterior probabilities annotations were calculated using TreeAnnotator in BEAST. An output tree was visualized in FigTree version 1.4.3.

Mechanical transmission to experimental herbaceous hosts. Leaf tissue ( $2 \mathrm{~g}$ ) from a symptomatic $C$. fruticosa plant was ground with $20 \mathrm{ml}$ of $0.05 \mathrm{M}$ phosphate buffer, $\mathrm{pH} 7.5$. The homogenate was used to mechanically inoculate the leaves of two groups of plants using carborundum. The first group of plants consisted of four Nicotiana benthamiana and four N. tabacum plants. For serial inoculations, TiRSaV-infected $N$. benthamiana and $N$. tabacum leaves were used as inoculum to mechanically inoculate six $N$. benthamiana and six $N$. tabacum plants, respectively. The second group consisted of seven plants each of cucumber [Cucumis sativus L. 'Boston Pickling'], watermelon [Citrullus lanatus (Thunb.) Matsum. \& Nakai 'Jubilee'], pumpkin [Cucurbita melo L. 'Connecticut Field'], squash [Cucurbita melo 'Dark Green Zucchini'], and pea [Pisum sativum L. 'Sugar Snap']. For both inoculation experiments, mock-inoculated plants (buffer only) were included as negative controls. The first group of plants were maintained in a growth room at $\sim 25^{\circ} \mathrm{C}$ and a $16-\mathrm{h} / 8-\mathrm{h}$ light/dark photoperiod for 1 to 3 months. The second group of plants were maintained in an air-conditioned glasshouse under natural light conditions. Inoculated plants were observed for symptom development and tested for TiRSaV infection on systemic leaves by RTPCR assays using the primer pairs TiRSaV-RdRp F/R and/or TiRSaV-NC F2/R2 following the protocols described above. Amplification products were cloned and sequenced as described above.

Detection of TiRSaV in eriophyid mites. The internal transcribed spacer (ITS) region and $28 \mathrm{~S}$ ribosomal RNA sequences and virus status of eriophyid mites recovered from symptomatic ti plants were determined following the approach of Druciarek et al. (2018). A single mite and a group of six eriophyid mites collected from a symptomatic ti leaf were placed into PCR tubes containing random primers $(5 \mu \mathrm{M})$ and dNTPs $(2 \mathrm{mM}$ each) in a volume of $14.5 \mu \mathrm{l}$. The mixture was heat-denatured at $90^{\circ} \mathrm{C}$ for $7 \mathrm{~min}$ and quickly chilled on ice for $2 \mathrm{~min}$. M-MLV-RT (160 u), rRNAsin $(28 \mathrm{u}$ ), and M-MLV-RT $1 \times$ buffer (Promega) were added to complete a final volume of $20 \mu \mathrm{l}$. The mixture was incubated at $37^{\circ} \mathrm{C}$ for $1 \mathrm{~h}$ and diluted fourfold, eightfold, and 16-fold. The primer sets MITS1 (5'-CCGTAGGTGAACCTGCGGAAGG-3')/MITS4 (5'-CCACCGT TAATTGTGATTTATTTTGTC-3') (Fenton et al. 1997; Kumar et al. 2001) and D1D2fw2 (5'-ACAAGTACCDTRAGGGAAAGTTG-3')/ 28Sr0990 (5'-CCTTGGTCCGTGTTTCAAGAC-3') (Mironov et al. 2012; Sonnenberg et al. 2007) were used in subsequent PCR reactions with $1 \mu l$ of each cDNA dilution as template. For TiRSaV detection, RT-PCR assays were performed using the primer pairs TiRSaV-RdRp F/R, TiRSaV-GP F1/R1, TiRSaV-NC F2/R2, TiRSaV-MP F1/R1, and TiRSaV-P8like F1/R1 as described above.

\section{Results}

Electron microscopy. In cells corresponding to the outer margin of the ringspot lesion, circular structures resembling DMBs approximately $85 \mathrm{~nm}$ in diameter were abundantly observed either directly in the cytoplasm or in cytoplasmic vesicles (Fig. 2) consistent with TSWV and emaravirus virions (Mielke-Ehret and Mühlbach 2012). These structures were primary observed in cells corresponding to the perimeter of the ringspot lesion, but not in cells of asymptomatic tissues.

Detection assays for TSWV and OFV. TSWV-specific LFAs and ELISAs were conducted on both symptomatic and asymptomatic ti leaf tissues. Both the LFAs and ELISAs were negative for all ti tissues tested. Both assays, however, were positive for TSWV-infected tomato and basil tissues that were used as a positive control (data not shown). RT-PCR using primers targeting a citrus strain of OFV (Roy et al. 2014) did not produce an amplification product. Although no positive control was available for this assay, all leaf samples tested were positive by RT-PCR using internal control primers targeting ti $r b c L$ mRNA, suggesting that the RNA was of suitable quality for amplification (data not shown).

Emaravirus and TiRSaV detection. The similarity in symptoms between ti ringspot and European mountain ash ringspot diseases, the presence of eriophyid mites on ti plants, as well as the presence of DMBs in symptomatic tissues for both of these diseases suggested that an emaravirus may play a role in ti ringspot disease. Samples from symptomatic plants were collected from the islands of Oahu, Maui, and Hawaii. Asymptomatic plant samples were collected from Oahu. Amplification products approximately $400 \mathrm{bp}$ in size were generated from the 10 symptomatic plants, but not the 10 asymptomatic plants (Fig. 3A). An amplification product of similar size was generated from the leaf sample of an edible fig (Ficus carica) infected with FMV, which served as a positive control for the assay. Amplification products were also generated from all ti leaf samples using primers targeting the ti $r b c L$, which served as an internal positive control for the assay (Fig. 3A). These primers, however, did not amplify any homolog(s) of $r b c L$ from the $F$. carica sample infected with FMV. No amplification products were generated with either RT-PCR assay when water was used as a template (Fig. 3A).

Eight of the 51 symptomatic samples did not produce the expected 213-bp product using primers RBC F3/L3 and were not assessed for the presence of TiRSaV as a result of potentially poor RNA or cDNA quality. Of the remaining 43 symptomatic samples, 40 (93\%) were 
positive for TiRSaV by the amplification of a 103-bp product. None of the 23 asymptomatic samples produced the 103-bp amplicon, indicating that they were negative for TiRSaV.

To determine whether a single emaravirus or multiple emaraviruses were present in symptomatic ti plants, degenerate-primer RTPCR (Elbeaino et al. 2013) amplification products from 13 samples collected from locations on Oahu, Maui, and Hawaii islands were cloned into pGEM-T Easy vector and sequenced. Amplification products from samples collected on all islands and locations were found to be $>97 \%$ identical, suggesting that only a single Emaravirus species was present in symptomatic ti plants.

Virus distribution in planta. To determine whether TiRSaV systemically infects common green ti, root, stem, petiole, and lamina from fully expanded symptomatic and unfurled asymptomatic leaf tissue from two plants underwent TiRSaV-specific RT-PCR as described above. Except for stem tissues, all tissues tested positive for TiRSaV infection, including asymptomatic leaf tissue, in both plants assayed (Fig. 3B).

Genome sequencing. HTS using an Illumina platform was performed on two libraries originating from different starting materials to draft the TiRSaV genome. The first library originated from a degenerate oligo-primed PCR library using dsRNA from symptomatic leaf tissue as template. A total of 490,503 single-end reads assembled into 3,036 contigs. Following database comparisons, it was determined that most of the viral contigs were derived from the velariviruses known to infect ti. Only three contigs shared highest similarity to emaraviruses and potentially the TiRSaV genome: two contigs (378 and $561 \mathrm{nt}$ ) corresponded to regions of the RdRp (RNA 1) and one contig (303 nt) corresponded to a region of the NC (RNA 3 ). The second library originated from RT-PCR amplification products utilizing a single primer targeting the conserved terminal sequence of emaraviruses. A total of 2,484,124 paired-end reads assembled into 1,952 contigs, of which six (970 to 2,399 nt) possessed significant homology to different Emaravirus genomic RNAs. These six contigs, when combined with the three contigs generated from the first library, and degenerate RT-PCR products produced a draft genome of TiRSaV consisting of five genomic RNAs designated as RNA1 to RNA5 (GenBank accessions MH223635 to MH223639). The viral termini of RNAs 1 to 5 were verified by RACE and both $5^{\prime}$ and $3^{\prime}$ ends of the three RNAs contained the 13 nt conserved in all emaraviruses. Virus-specific primers were used to bridge sequence gaps and clarify ambiguous regions. Very high depth of coverage was achieved for RNAs 2 to 5, but not RNA 1 (Table 1). To complete the sequence of RNA 1 (RdRp), degenerate primers targeting conserved motifs $\mathrm{A}$ and $\mathrm{C}$ of the RdRp (Elbeaino et al. 2013) and other internal conserved regions (EMARA $F$ and R7 5'-TCTTGTGGTGATCCATGIARRCCYTTATTWCC-3', EMARA $F$ and R8 5'-CCGCGCAGATAATCTTATARAIG AYAARYTRGAAT-3') (Olmedo-Velarde, unpublished) were used in conjunction with TiRSaV RNA 1-specific primers to bridge the
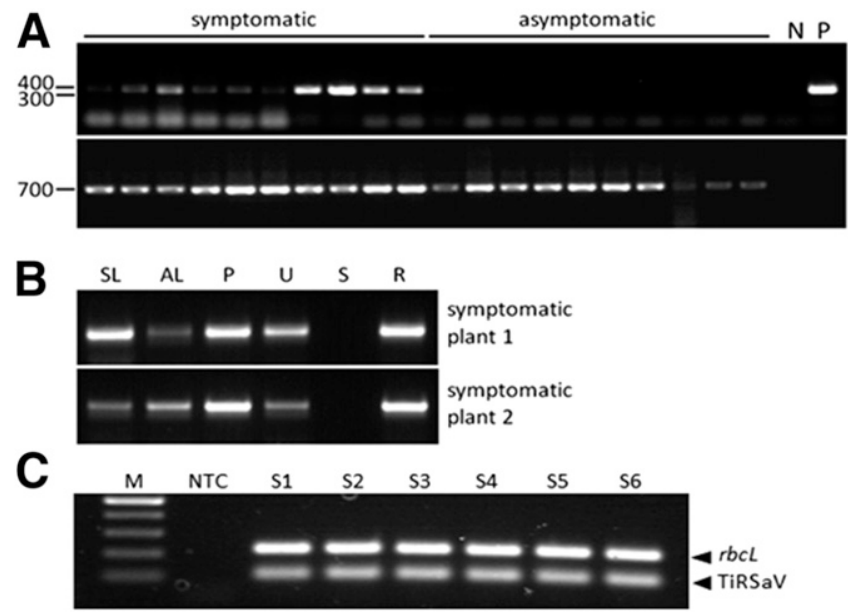

Fig. 3. Emaravirus and ti ringspot-associated virus (TiRSaV) detection in Cordyline fruticosa plants affected by ti ringspot disease using degenerate-primer and virusspecific reverse transcription PCR (RT-PCR), respectively. A, Association of Emaravirus infection with ti ringspot disease symptoms. The upper panel shows Emaravirus detection in symptomatic but not asymptomatic ti plants. The lower panel shows detection of $C$. fruticosa rubisco large subunit $(r b c L)$ as an indication of cDNA quality. Numbers on the left indicate size in base pairs. $\mathrm{N}=$ water control and $\mathrm{P}=$ positive control (Fig mosaic emaravirus). $\mathbf{B}$, Emaravirus sequences were detected in all but stem tissues of two ti plants with symptoms of ti ringspot disease. $\mathrm{SL}=$ symptomatic leaf lamina, $\mathrm{AL}=$ asymptomatic leaf lamina, $\mathrm{P}=$ petiole of symptomatic leaf, $U=$ unfurled leaf lamina, $S=$ stem, and $R=$ root. $C, A$ multiplex RT-PCR assay for the specific detection of TiRSaV. TiRSaV was amplified in symptomatic samples S1 to S6 (103-bp amplicon) but not from a nontemplate control (NTC). Primers targeting a 213-bp region of the $r b c L$ of $C$. fruticosa served as an internal positive control for the assay. $M=100-b p$ DNA ladder.
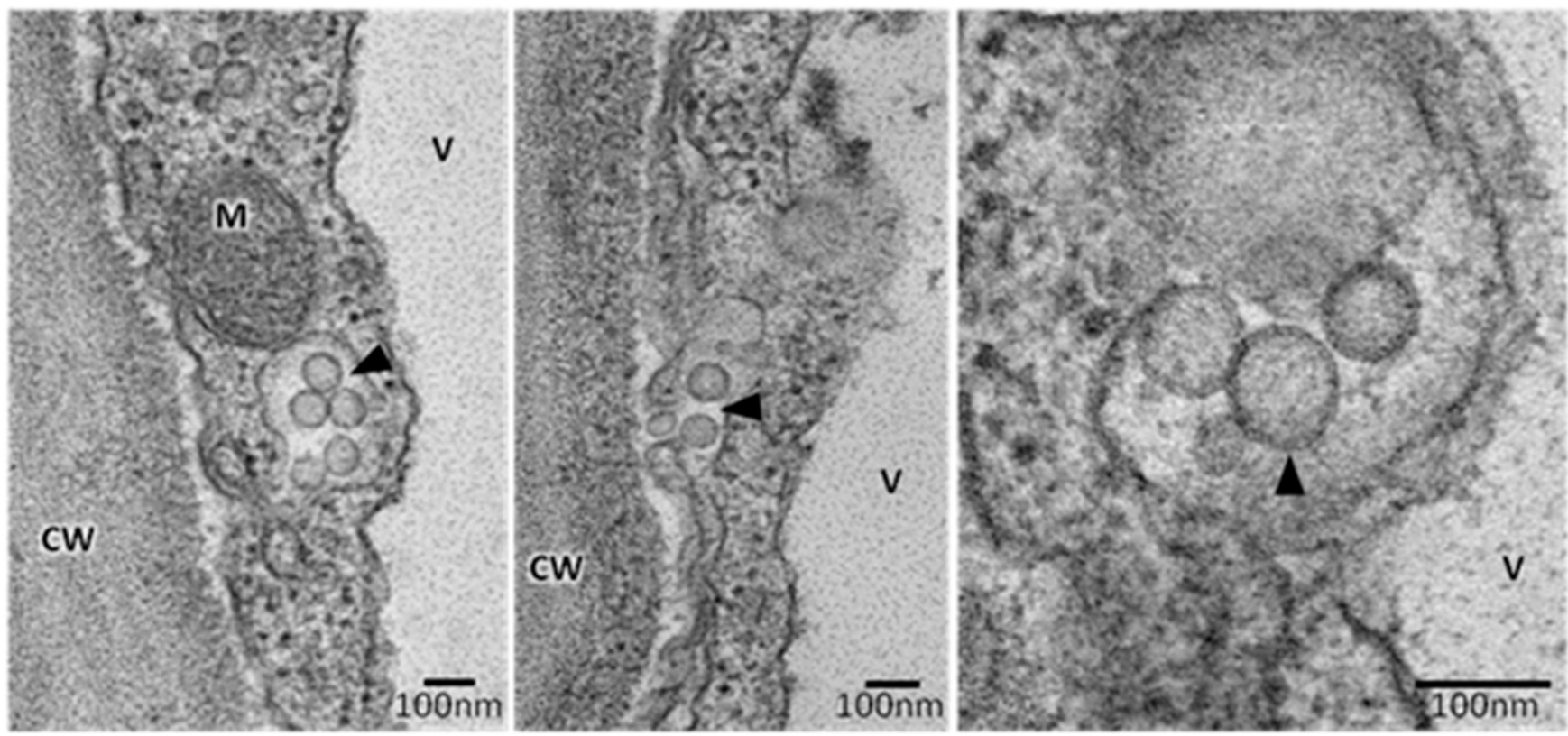

Fig. 2. Transmission electron micrographs showing double membrane bodies approximately $85 \mathrm{~nm}$ in diameter (arrowheads) present in the cells of common green ti plants (Cordyline fruticosa) displaying symptoms of ti ringspot disease. $\mathrm{CW}=$ cell wall, $\mathrm{M}=$ mitochondrion, and $\mathrm{V}=$ vacuole. 
sequence gaps. The five RNAs were present only in symptomatic plants as determined by RT-PCR assays using TiRSaV RNAspecific primers with total RNA from seven symptomatic plants and seven asymptomatic plants used as template (Supplementary Fig. S1), indicating that these five RNAs were part of the TiRSaV genome and further associating this virus as the causal agent of the disease. Further screening for additional TiRSaV RNAs was undertaken by searching for contigs with the following features: were $>100 \mathrm{nt}$ in length, had AT-rich $5^{\prime}$ and $3^{\prime}$ untranslated regions between 100 and $500 \mathrm{nt}$ in length (if present), composed of a high read count ( $\mathrm{Lu}$ et al. 2015), and encoded a protein with no homology in the GenBank database. One candidate contig was identified. However, RT-PCR with primers specific to this contig demonstrated that it was present in both symptomatic and asymptomatic ti plants, suggesting that the contig was not an additional TiRSaV RNA (data not shown).

Genome organization and phylogenetic analyses. Five negativesense RNA segments predicted to encode proteins with homology to emaraviruses were found to comprise the TiRSaV genome, with each RNA segment encoding a single ORF (Fig. 4) on the complementary strand. TiRSaV RNA 1 was 7,217 nt in length with one ORF (ORF1, CAU7161-UAA139) coding for a putative RdRp (p1) of 2,340 amino acids (aa) and had a predicted molecular mass of $272 \mathrm{kDa}$. Pairwise comparisons of the deduced RdRp sequence revealed identities with orthologous proteins of emaraviruses ranging from 37 to $45 \%$ (Table 2). TiRSaV RNA 2 was 2,399 nt in length with one ORF (ORF2, CAU2341-CUA188) coding for a putative GP (p2) of 717

Table 1. Number of high-throughput sequencing reads and depth of coverage values for the five RNAs that comprise the ti ringspot-associated virus (TiR$\mathrm{SaV}$ ) genome

\begin{tabular}{lccrr}
\hline \multirow{2}{*}{$\begin{array}{l}\text { TiRaV RNA } \\
\text { (nucleotide length) }\end{array}$} & No. of reads & \multicolumn{3}{c}{ Depth of coverage (no. of reads) } \\
\cline { 3 - 5 } & Minimum & \multicolumn{1}{c}{ Mean } & Maximum \\
\hline RNA 1 $(7,217)$ & 9,556 & 0 & 261 & 2,086 \\
RNA 2 $(2,399)$ & 590,742 & 44 & 43,325 & 77,253 \\
RNA 3 $(1,106)$ & 663,446 & 16 & 118,444 & 168,139 \\
RNA 4 $(1,342)$ & 56,758 & 1 & 9,023 & 14,633 \\
RNA 5 $(1,323)$ & 32,044 & 8 & 3,841 & 12,979 \\
\hline
\end{tabular}

aa and had a predicted molecular mass of $83 \mathrm{kDa}$. The GP shared identities with orthologs from emaraviruses ranging from 23 to $38 \%$ (Table 2). TiRSaV RNA 3 was 1,106 nt in length encoding one ORF (ORF3, CAU978-UUA118) coding for a putative NC (p3) of 286 aa and had a predicted molecular mass of $32 \mathrm{kDa}$. The NC shared identity levels of 28 to $39 \%$ with the orthologs of emaraviruses (Table 2). TiRSaV RNA 4 was found to be 1,342 nt in length with a single ORF (ORF4, CAU1261-UUA263) coding for a putative MP (p4) of 332 aa and had a predicted molecular mass of $38 \mathrm{kDa}$. The identity levels with orthologs of emaraviruses ranged from 20 to $51 \%$ (Table 2). TiRSaV RNA 5 was 1,323 nt in length and found to encode one ORF (ORF5, CAU1254-CUA739) coding for a putative protein with unknown function (p5) of 171 aa and had a predicted molecular mass of $21 \mathrm{kDa}$. The identity levels with orthologs from emaraviruses ranged from 29 to $32 \%$ (Table 2). The putative TiRSaV proteins $\mathrm{p} 1$, p2, p3, and p5 shared the highest identity levels with orthologs from RLBV, ranging from 32 to $45 \%$. In contrast, the putative TiRSaV p4 shared the highest identity level (51\%) with the HPWMoV ortholog (Table 2). Phylogenetic analyses were performed with two characterbased algorithms (maximum likelihood and Bayesian inference) employing $\mathrm{LG}+\mathrm{G}$ as the best model of protein evolution. Both algorithms predicted similar relationships between members and putative members of the genus Emaravirus. Three distinct clades with similar topologies were formed with both methods and in the four phylogenetic trees: one clade was composed of FMV, PPSMV 2, RRV, BLMaV, and PPSMV 1; the next clade was composed of AcCRaV, EMARaV, and RYRSaV; and the third clade was composed of HPWMoV, PVBV, RLBV, and TiRSaV (Fig. 5).

Mechanical transmission to experimental herbaceous hosts. Around 30 days postinoculation (dpi), systemic leaves from all inoculated plants in the first group were tested by RT-PCR using the specific primer set TiRSaV-RdRp F/R. PCR products of expected size were obtained from two $N$. benthamiana plants and two N. tabacum plants (Supplementary Fig. S2). Sequencing of the amplicons confirmed the identity of TiRSaV showing the partial fragment of TiRSaV RNA 1 from both $N$. benthamiana and $N$. tabacum plants sharing 99\% identity to the TiRSaV RNA 1 from $C$. fruticosa, thus demonstrating mechanical transmission of TiRSaV to experimental herbaceous hosts. Both $N$. benthamiana plants remained asymptomatic for 3 months, but one $N$. tabacum plant presented mild veinal

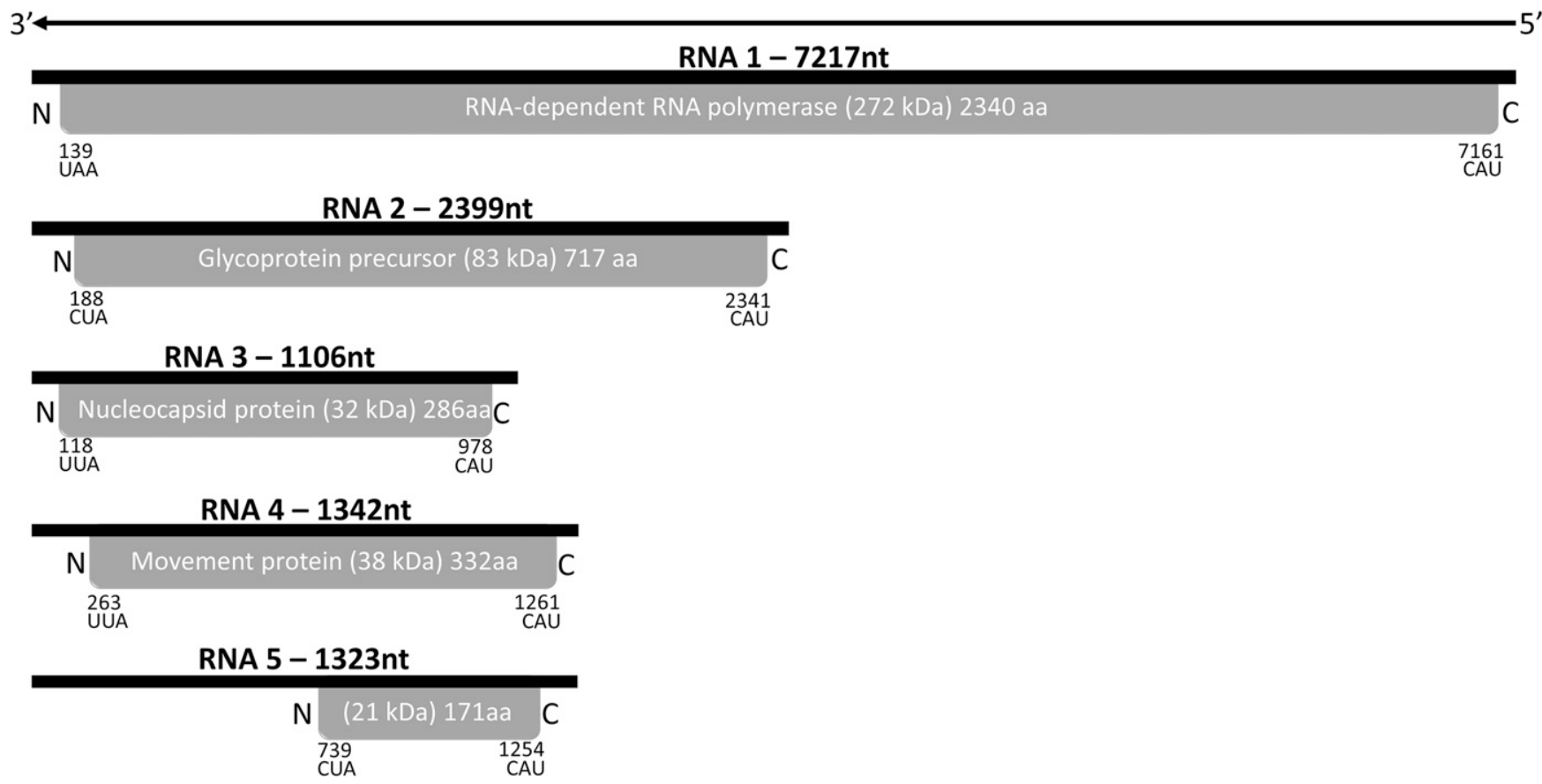

Fig. 4. Genome organization of ti ringspot-associated virus. Shaded boxes represent the predicted open reading frame (ORF) for each RNA. The putative protein product for each ORF, start and stop codons, function (if known), and estimated molecular weight are provided. For the purpose of clarity, the genomic RNAs are not drawn to scale. 
chlorosis at 30 dpi (Supplementary Fig. S3). All six N. benthamiana plants and three of the six $N$. tabacum plants serially inoculated with TiRSaV tested positive for the virus using the specific primer set TiRSaV-NC F2/R2 that amplifies a region of the NC (RNA 3). Sequencing of the amplicons confirmed that TiRSaV can be mechanically transmissible to $N$. benthamiana and $N$. tabacum, eliciting mild veinal chlorosis in some of $N$. tabacum plants. In the second group of plants, amplification products of the expected size were obtained from three cucumber plants, one watermelon plant, one pumpkin plant, and one squash plant (data not shown). In all of the RT-PCR assays, the nontemplate control and mock-inoculated plants tested negative. All inoculated plants remained asymptomatic during 3 months of observation.

Eriophyid mites and detection of TiRSaV. Primer sets MITS1/ MITS4 (ITS region) and D1D2fw2/28Sr0990 (28S region) generated amplicons of $\sim 620$ and $\sim 640 \mathrm{bp}$, respectively. For both primer sets, amplicons were obtained only when cDNA synthesized from six mites was used as starting material (Supplementary Fig. S4). After cloning and sequencing four clones of each amplicon, a BLAST search revealed that the ITS sequence (MH796797) was 83\% identical to the ITS region from Aceria parapopuli (KF782488) with a query coverage of only $41 \%$. For the $28 \mathrm{~S}$ rDNA region, two groups of sequences were obtained, sharing only $77 \%$ of identity between each other. One group (MH796799) shared 87\% identity to Acaphyllisa sp. (KF782488) and Neolitaculus sp. (KM111069), whereas the other group (MH796800) shared 88\% identity to Calacarus carinatus (KM111063), both with complete query coverage.

Using cDNA from the group of six mites diluted fourfold, RTPCR products of the correct size were generated for four of the five TiRSaV RNAs. Only RNA 2 encoding the glycoprotein was not detected. This RT-PCR detection of TiRSaV indicates ingestion and perhaps acquisition of the virus by eriophyid mites.

\section{Discussion}

The common green ti variety was introduced into Hawaii centuries ago by early Polynesian voyagers. Its pollen and seeds are not viable (Hinkle 2007) and ti has therefore been propagated exclusively by vegetative cuttings since its introduction, making it prone to infection by systemic pathogens such as viruses. Indeed, individual ti plants can be coinfected with at least four species in the genus Velarivirus and ti is also a host to TSWV and OFV (Cho et al. 1986; Dietzgen et al. 2018; Melzer et al. 2013b). None of these velariviruses, however, were associated with ti ringspot (Melzer et al. 2013a). In this

Table 2. Percent amino acid identities between orthologous proteins of ti ringspot-associated virus (TiRSaV) and members and putative members of the genus Emaravirus ${ }^{\mathrm{a}}$

\begin{tabular}{lccccc}
\hline & \multicolumn{5}{c}{ TiRSaV protein } \\
\cline { 2 - 6 } Emaravirus species & p1 & p2 & $\mathbf{p 3}$ & $\mathbf{p 4}$ & $\mathbf{p 5}^{\mathbf{b}}$ \\
\hline RLBV & 45 & 38 & 39 & 48 & 32 \\
HPWMoV & 44 & 36 & 37 & 51 & 29 \\
BPVBV & 44 & 31 & 33 & 50 & - \\
FMV & 39 & 23 & 33 & 20 & - \\
EMARaV & 38 & 24 & 29 & - & - \\
RYRSaV & 38 & 26 & 31 & 22 & - \\
AcCRaV & 38 & 27 & 29 & 22 & - \\
BLMaV & 38 & 26 & 34 & 20 & - \\
PPSMV 2 & 38 & 24 & 34 & 21 & - \\
RRV & 37 & 25 & 31 & 23 & - \\
PPSMV 1 & 37 & 25 & 28 & 23 & - \\
\hline
\end{tabular}

${ }^{a}$ RLBV $=$ Raspberry leaf blotch emaravirus, $\mathrm{HPWMoV}=$ High Plains wheat mosaic emaravirus, BPVBV = blue palo verde broom virus, FMV = Fig mosaic emaravirus, $\mathrm{EMARaV}=$ European mountain ash ringspot-associated emaravirus, RYRSaV = Redbud yellow ringspot-associated emaravirus, $\mathrm{AcCRaV}=$ Actinidia chlorotic ringspot-associated emaravirus, $\mathrm{BLMaV}=$ blackberry leaf mottle-associated virus, PPSMV 1 and 2, Pigeon pea sterility mosaic emaravirus 1 and $2, \mathrm{RRV}=$ Rose rosette emaravirus.

${ }^{b}$ The orthologs of TiRSaV p5 have been designated $\mathrm{p} 8$ for HPWMoV and RLBV. Dashes indicate no homologous proteins have been identified for these viruses.
A
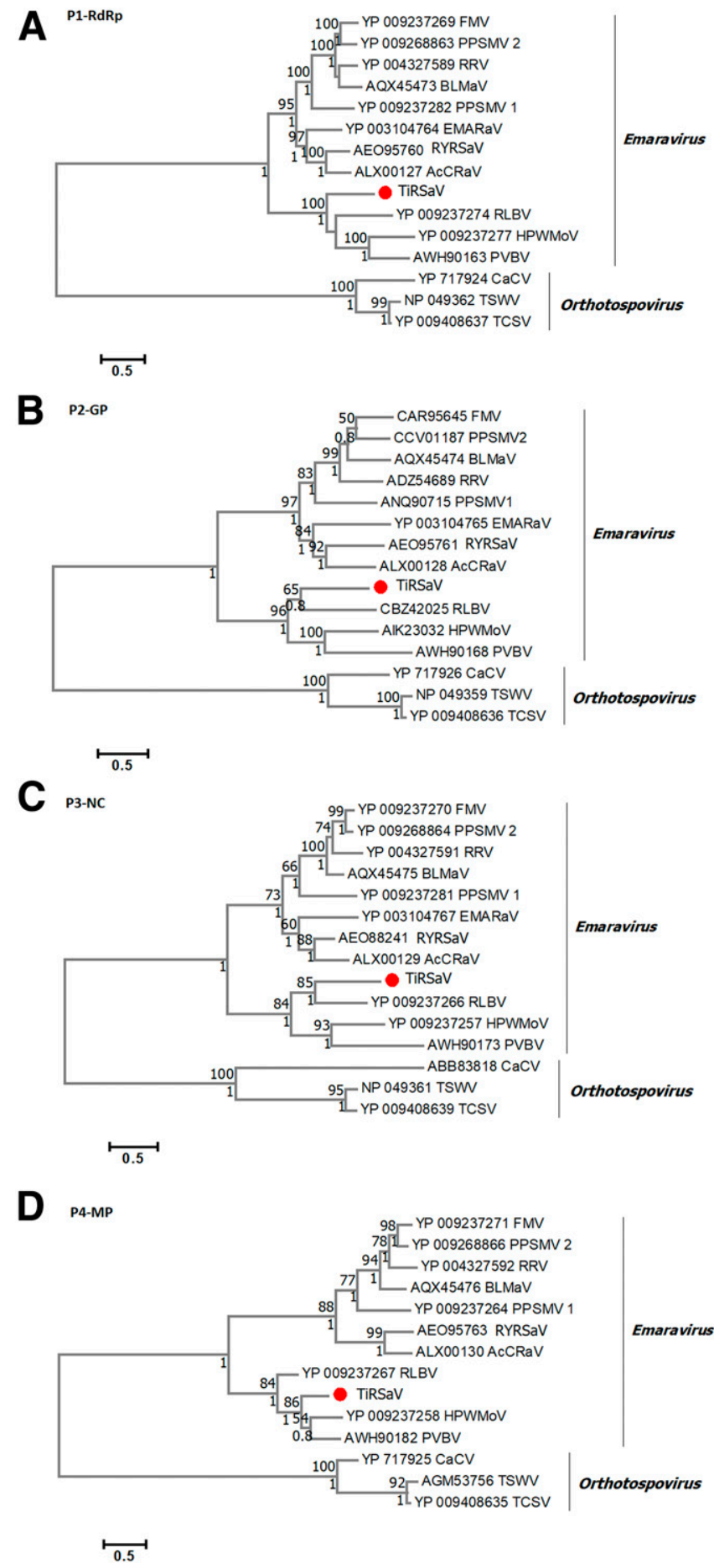

Fig. 5. Phylogenetic placement of ti ringspot-associated virus (TiRSaV) within the genus Emaravirus using the A, RNA-dependent RNA polymerase (RdRp), B, glycoprotein precursor (GP), C, nucleocapsid (NC), and D, movement protein (MP) sequences. The phylogenetic trees were generated using the maximum likelihood method with 1,000 bootstrap values to estimate branch support. The numbers above the nodes represent bootstrap values $>50$, and those under the node represent posterior probabilities $>0.7$ generated with Bayesian inference with three Markov chains Monte Carlo and $10,000,000$ generations per chain. The scale represents the number of substitutions per unit branch length. Capsicum chlorosis orthotospovirus (CaCV), tomato spotted wilt tospovirus (TSWV), and tomato chlorotic spot tospovirus (TCSV) were used as an outgroup. The GenBank accession numbers for each sequence used in the analyses are provided. FMV = Fig mosaic emaravirus, PPSMV 1 and $2=$ Pigeon pea sterility mosaic emaravirus 1 and 2, RRV = Rose rosette emaravirus, BLMaV = blackberry leaf mottle-associated virus, EMARaV = European mountain ash ringspot-associated emaravirus, RYRSaV = Redbud yellow ringspot-associated emaravirus, AcCRaV = Actinidia chlorotic ringspot-associated emaravirus, RLBV = Raspberry leaf blotch emaravirus, HPWMoV = High Plains wheat mosaic emaravirus, and PVBV = blue palo verde broom virus. 
study, we demonstrate that TSWV and OFV are also not associated with the disease, as these two pathogens could not be detected using serological and/or molecular assays. The presence of eriophyid mites on ti plants, DMBs in the cytoplasm of symptomatic tissues (Fig. 2), and the similarity in symptoms between ti ringspot and those caused by or associated with Emaravirus infection (Fig. 1) led to the testing of symptomatic ti plants for the presence of an emaravirus. The generation of an amplification product from symptomatic tissue using a universal Emaravirus RT-PCR assay indicated that at least one emara-like virus species was present in the symptomatic tissue evaluated (Fig. 3). The cloning and sequencing of this product suggested that the virus was an undescribed Emaravirus species because of its low sequence identity with sequences in GenBank. Since a host plant species can be infected by multiple Emaravirus species that cause similar symptoms (e.g., PPSMV 1 and PPSMV 2 on Cajanus cajan) (Elbeaino et al. 2015; Kumar et al. 2003), the sequence of the universal Emaravirus RT-PCR product was obtained for 13 isolates from the islands of Oahu, Maui, and Hawaii. These sequences indicated there was minimal sequence variation between isolates, suggesting that only a single virus species was associated with the disease. This observation was generally supported by the development of a specific RT-PCR assay that detected the virus in 40 of 43 symptomatic ti plants. It is possible that the three symptomatic plants that tested negative using the RT-PCR assay were infected by a TiRSaV variant that could not be detected using the current detection primers. It is also possible that low virus titer, uneven virus distribution ( $\mathrm{Lu}$ et al. 2015), different relative frequency of each emaraviral segment in different scenarios as seen in other multipartite viruses (SanchezNavarro et al. 2013; Sicard et al. 2013), or technical challenges can explain this discrepancy. Regardless, both universal Emaravirus and virus-specific RT-PCR assays strongly associated the presence of this virus with ti ringspot symptoms. We have therefore proposed the name TiRSaV for this ti pathogen.

The DMBs observed by transmission electron microscopy were predominantly found in the cytoplasm of cells within the chlorotic zone of the ringspot lesion. Outside of this zone, DMBs were infrequent. This observation, in conjunction with the lack of obvious symptoms in newly unfurled leaves, suggested that TiRSaV was not a systemic pathogen and instead the ringspots were a local lesion caused by infection from viruliferous vector feeding in a manner similar to citrus leprosis (Rodrigues et al. 2003). A nonsystemic infection has also been suggested for RYRSaV in the redbud (Cersis canadensis) variety Forest Pansy (Di Bello et al. 2016). RT-PCR, however, clearly indicated that $\mathrm{TiRSaV}$ was systemic owing to its presence in all tissues sampled aside from the woody stem tissues (Fig. 3B). The inability to detect the virus in the stem tissue may be technical or attributable to low virus titer. It is unclear why new leaves emerge without symptoms but invariably develop ringspots in the weeks following unfurling. We have observed that newly emerged leaves of $F$. carica plants in Hawaii are often free of symptoms of fig mosaic, whereas mature leaves display symptoms, suggesting that the delayed onset of foliar symptoms is not unique to ti ringspot.

In the first approach to characterize the genome of TiRSaV, HTS of a dsRNA library generated by a shotgun approach derived from the symptomatic ti plant produced relatively little sequence information on the new virus. Conversely, HTS generated a large amount of sequence information on the previously characterized velariviruses that coinfected the plant. This may be a reflection on the relative titers of velariviruses and emaraviruses in ti plants but may also be attributable to different amounts of dsRNA produced by +ssRNA and ssRNA viruses during replication and/or transcription. However, in the second approach, HTS performed on PCR products generated using the Emaravirus conserved terminal primer together with conventional sequencing allowed the determination of the complete sequences of four of the five segments of the TiRSaV genome, whose molecular features are consistently related to those of emaraviruses. The inability of different polymerases to amplify the complete sequence of the RdRp of emaraviruses using the Emaravirus consensus terminal primer was previously reported (Hassan et al. 2017), so the use of degenerate primers located in Emaravirus RdRp motifs and partial fragments generated by the shotgun library addressed this issue and allowed the complete sequence of RNA 1 to be obtained. The phylogenetic relationships inferred from the protein sequences of the emaraviruses, putative emaraviruses, and $\mathrm{TiRSaV}$ clearly place the latter within the genus Emaravirus, specifically in a clade with RLBV, HPWMoV, and PVBV (Fig. 4). Four of the TiRSaV proteins (p1, p2, p3, and p5) showed the highest homology to RLBV, while p4 showed the highest homology to HPWMoV (Fig. 5; Table 2).

The genomes of RLBV and HPWMoV have both been reported to consist of eight genomic segments (Lu et al. 2015; McGavin et al. 2012; Tatineni et al. 2014). These viruses belong to a phylogenetically distinct clade in the genus (Fig. 5) and are characterized by the presence of the four core segments and an additional four segments coding for putative proteins with unknown function and no apparent homology with other emaravirus proteins. However, the TiRSaV genome consisted of the four core segments of the emaraviruses and an additional segment coding for putative protein with unknown function and similarity to the p8 of RLBV and HPWMoV. Attempts to identify additional TiRSaV genome segments using RT-PCR and a deep exploration in the HTS dataset failed, but we cannot exclude the existence of additional TiRSaV segments as other additional segments in other emaraviruses remained elusive until further studies were undertaken (Di Bello et al. 2016; Elbeaino et al. 2012; Lu et al. 2015). PVBV is a putative emaravirus infecting blue palo verde trees in Arizona, likely to be vectored by the eriophyid mite Aculus cercidi, and is closely associated with witches' broom disease of the trees. This virus has been reported to have four RNA segments coding for the core proteins of emaraviruses that show the highest homology to HPWMoV proteins (Ilyas et al. 2018). Both PVBV and TiRSaV were characterized using HTS approaches and belong to the same clade in the phylogeny of emaraviruses. This may suggest that the four core segments of emaraviruses are enough to cause disease in their hosts and additional segments may help to enhance virulence or pathogenicity.

A lack of consistent homology has been reported among the proteins $\mathrm{p} 5$ to $\mathrm{p} 8$ coded by emaraviruses (Hassan et al. 2017; Tatineni et al. 2014), suggesting that some emaraviruses have more than eight RNA segments (Stewart 2016). Gupta et al. (2018) reported that HPWMoV p7 and p8 are suppressors of RNA silencing. In contrast, $\mathrm{Lu}$ et al. (2015) demonstrated that RLBV p6 and p7 are related to RLBV pathogenicity, although they could not detect RNA silencing suppression in RLBV p2 to p8. Further characterization of the function of these putative proteins may help researchers better understand the evolution of emaraviruses and the apparent discrepancy in the number of genomic RNA segments between species.

Eriophyid mites have been identified as the vectors of other emaraviruses (Caglayan et al. 2012; McGavin et al. 2012; Mielke-Ehret et al. 2010). In Hawaii, both symptomatic and asymptomatic ti plants have been found to be infested with these mites. A preliminary molecular examination of mites collected in this study suggests that at least two species coexist on symptomatic leaves; based on the low sequence identities between them and with current accessions in GenBank, both may represent previously undescribed species. Four of five segments of the TiRSaV genome were detected in this mixed sample, making these mites candidates for future taxonomic and virus transmission efforts.

TiRSaV was successfully transmitted mechanically to multiple experimental herbaceous plants including Nicotiana and cucurbit species. Zheng et al. (2017) were similarly able to mechanically transmit AcCRaV to $N$. benthamiana, although not all AcCRaV-infected plants showed symptoms. The ability of TiRSaV to systemically infect these experimental hosts will facilitate any future virus purification experiments for the purpose of antibody production.

Since the first report of ti ringspot disease in 2009, no causal agent responsible for the disease has been identified. In this study, we describe a new virus species that is associated with the disease. At present, ti ringspot has not been reported outside of Hawaii. Since ti is a globally popular ornamental grown both indoors and outdoors, the diagnostic assays implemented to detect the virus and its RNA segments are critical for preventing the further 
spread of the disease in Hawaii and other locations where the disease is not yet present.

\section{Literature Cited}

Afgan, E., Baker, D., van den Beek, M., Blankenberg, D., Bouvier, D., Čech, M., Chilton, J., Clements, D., Coraor, N., Eberhard, C., Grüning, B., Guerler, A., Hillman-Jackson, J., Von Kuster, G., Rasche, E., Soranzo, N., Turaga, N., Taylor, J., Nekrutenko, A., and Goecks, J. 2016. The Galaxy platform for accessible, reproducible and collaborative biomedical analyses: 2016 update. Nucleic Acids Res. 44:W3-W10.

Altschul, S. F., Madden, T. L., Schäffer, A. A., Zhang, J., Zhang, Z., Miller, W., and Lipman, D. J. 1997. Gapped BLAST and PSI-BLAST: A new generation of protein database search programs. Nucleic Acids Res. 25:3389-3402.

Bi, Y., Tugume, A. K., and Valkonen, J. P. T. 2012. Small-RNA deep sequencing reveals Arctium tomentosum as a natural host of Alstroemeria virus $X$ and a new putative emaravirus. PLoS One 7:e42758.

Bolger, A. M., Lohse, M., and Usadel, B. 2014. Trimmomatic: A flexible trimmer for Illumina sequence data. Bioinformatics 30:2114-2120.

Bouckaert, R., Heled, J., Kühnert, D., Vaughan, T., Wu, C.-H., Xie, D., Suchard, M. A., Rambaut, A., and Drummond, A. J. 2014. BEAST 2: A software platform for Bayesian evolutionary analysis. PLoS Comput. Biol. 10:e1003537.

Caglayan, K., Elci, E., Ulubas Serce, C., Kaya, K., Gazel, M., and Medina, V. 2012. Detection of fig mosaic virus in viruliferous eriophyid mite Aceria ficus. J. Plant Pathol. 94:629-634.

Cho, J. J., Mau, R. F. L., Gonsalves, D., and Mitchell, W. C. 1986. Reservoir weed hosts of tomato spotted wilt virus. Plant Dis. 70:1014-1017.

Di Bello, P. L., Laney, A. G., Druciarek, T., Ho, T., Gergerich, R. C., Keller, K. E., Martin, R. R., and Tzanetakis, I. E. 2016. A novel emaravirus is associated with redbud yellow ringspot disease. Virus Res. 222:41-47.

Dietzgen, R. G., Tassi, A. D., Freitas-Astúa, J., and Kitajima, E. W. 2018. First report of orchid fleck virus and its mite vector on green cordyline. Australas. Plant Dis. Notes 13:11.

Druciarek, T., Lewandowski, M., and Tzanetakis, I. 2018. Direct RT-PCR assay for virus detection and eriophyoid species identification. Poster presented at: International Congress of Plant Pathology 2018, Plant Health in a Global Economy; 29 July to 3 August 2018; Boston, MA.

Elbeaino, T., Digiaro, M., and Martelli, G. P. 2012. RNA-5 and -6, two additional negative-sense RNA segments associated with fig mosaic virus. J. Plant Pathol. 94:421-425.

Elbeaino, T., Digiaro, M., Mielke-Ehret, N., Muehlbach, P., and Martelli, G. P. 2018. ICTV virus taxonomy profile: Fimoviridae. J. Gen. Virol. 99:1478-1479.

Elbeaino, T., Digiaro, M., Uppala, M., and Sudini, H. 2015. Deep sequencing of dsRNAs recovered from mosaic-diseased pigeonpea reveals the presence of a novel emaravirus: Pigeonpea sterility mosaic virus 2. Arch. Virol. 160: 2019-2029.

Elbeaino, T., Whitfield, A., Sharma, M., and Digiaro, M. 2013. Emaravirusspecific degenerate PCR primers allowed the identification of partial RNAdependent RNA polymerase sequences of Maize red stripe virus and Pigeonpea sterility mosaic virus. J. Virol. Methods 188:37-40.

Ewels, P., Magnusson, M., Lundin, S., and Käller, M. 2016. MultiQC: Summarize analysis results for multiple tools and samples in a single report. Bioinformatics 32:3047-3048.

Fenton, B., Malloch, G., and Moxey, E. 1997. Analysis of eriophyid mite rDNA internal transcribed spacer sequences reveals variable simple sequence repeats. Insect Mol. Biol. 6:23-32.

Grabherr, M. G., Haas, B. J., Yassour, M., Levin, J. Z., Thompson, D. A., Amit, I., Adiconis, X., Fan, L., Raychowdhury, R., Zeng, Q., Chen, Z., Mauceli, E., Hacohen, N., Gnirke, A., Rhind, N., di Palma, F., Birren, B. W., Nusbaum, C., Lindblad-Toh, K., Friedman, N., and Regev, A. 2011. Full-length transcriptome assembly from RNA-Seq data without a reference genome. Nat. Biotechnol. 29:644-652.

Gupta, A. K., Hein, G. L., Graybosch, R. A., and Tatineni, S. 2018. Octapartite negative-sense RNA genome of High Plains wheat mosaic virus encodes two suppressors of RNA silencing. Virology 518:152-162.

Hassan, M., Di Bello, P. L., Keller, K. E., Martin, R. R., Sabanadzovic, S., and Tzanetakis, I. E. 2017. A new, widespread emaravirus discovered in blackberry. Virus Res. 235:1-5

Hinkle, A. E. 2007. Population structure of Pacific Cordyline fruticosa (Laxmanniaceae) with implications for human settlement of Polynesia. Am. J. Bot. 94:828-839.

Huang, X., and Miller, W. 1991. A time-efficient, linear-space local similarity algorithm. Adv. Appl. Math. 12:337-357.

Ilyas, M., Avelar, A. S., Schuch, U., and Brown, J. K. 2018. First report of an emaravirus associated with witches broom disease and eriophyid mite infestations of the blue palo verde tree in Arizona. Plant Dis. 102:1863.

Kearse, M., Moir, R., Wilson, A., Stones-Havas, S., Cheung, M., Sturrock, S., Buxton, S., Cooper, A., Markowitz, S., Duran, C., Thierer, T., Ashton, B.,
Meintjes, P., and Drummond, A. 2012. Geneious Basic: An integrated and extendable desktop software platform for the organization and analysis of sequence data. Bioinformatics 28:1647-1649.

Kumar, P. L., Fenton, B., Duncan, G., Jones, A., Sreenivasulu, P., and Reddy, D. 2001. Assessment of variation in Aceria cajani using analysis of rDNA ITS regions and scanning electron microscopy: Implications for the variability observed in host plant resistance to pigeonpea sterility mosaic disease. Ann. Appl. Biol. 139:61-73.

Kumar, P. L., Jones, A. T., and Reddy, D. V. R. 2003. A novel mite-transmitted virus with a divided RNA genome closely associated with pigeonpea sterility mosaic disease. Phytopathology 93:71-81.

Kumar, S., Stecher, G., and Tamura, K. 2016. MEGA7: Molecular Evolutionary Genetics Analysis version 7.0 for bigger datasets. Mol. Biol. Evol. 33: 1870-1874.

Langmead, B., Trapnell, C., Pop, M., and Salzberg, S. L. 2009. Ultrafast and memory-efficient alignment of short DNA sequences to the human genome. Genome Biol. 10:R25.

Lu, Y., McGavin, W., Cock, P. J. A., Schnettler, E., Yan, F., Chen, J., and MacFarlane, S. A. 2015. Newly identified RNAs of raspberry leaf blotch virus encoding a related group of proteins. J. Gen. Virol. 96:3432-3439.

McGavin, W. J., Mitchell, C., Cock, P. J. A., Wright, K. M., and MacFarlane, S. A. 2012. Raspberry leaf blotch virus, a putative new member of the genus Emaravirus, encodes a novel genomic RNA. J. Gen. Virol. 93:430-437.

Melzer, M., Ayin, C., Sugano, J., Uchida, J., Kawate, M., Borth, W., and Hu, J. 2013a. Differentiation and distribution of Cordyline viruses 1-4 in Hawaiian ti plants (Cordyline fruticosa L.). Viruses 5:1655-1663.

Melzer, M. J., Sether, D. M., Borth, W. B., Mersino, E. F., and Hu, J. S. 2011. An assemblage of closteroviruses infects Hawaiian ti (Cordyline fruticosa L.). Virus Genes 42:254-260

Melzer, M. J., Sugano, J. S., Uchida, J. Y., Borth, W. B., Kawate, M. K., and Hu, J. S. 2013b. Molecular characterization of closteroviruses infecting Cordyline fruticosa L. in Hawaii. Front. Microbiol. 4:39.

Mielke-Ehret, N., and Mühlbach, H.-P. 2012. Emaravirus: A novel genus of multipartite, negative strand RNA plant viruses. Viruses 4:1515-1536.

Mielke-Ehret, N., Thoma, J., Schlatermund, N., and Mühlbach, H. P. 2010. Detection of European mountain ash ringspot-associated virus-specific RNA and protein P3 in the pear leaf blister mite Phytoptus pyri (Eriophyidae) Arch. Virol. 155:987-991.

Mironov, S. V., Dabert, J., and Dabert, M. 2012. A new feather mite species of the genus Proctophyllodes Robin, 1877 (Astigmata: Proctophyllodidae) from the long-tailed tit Aegithalos caudatus (Passeriformes: Aegithalidae)-morphological description with DNA barcode data. Zootaxa 3253:54-61.

Morris, T. J., and Dodds, J. A. 1979. Isolation and analysis of double-stranded RNA from virus-infected plant and fungal tissue. Phytopathology 69:854-858.

Mühlbach, H.-P., and Mielke-Ehret, N. 2012. Emaravirus. Pages 767-769 in: Virus Taxonomy: Classification and Nomenclature of Viruses, Ninth Report of the International Committee on Taxonomy of Viruses. A. M. Q. King, M. J. Adams, E. B. Carstens, and E. J. Lefkowitz, eds. Elsevier Academic Press, London, UK.

Rodrigues, J. C. V., Kitajima, E. W., Childers, C. C., and Chagas, C. M. 2003. Citrus leprosis virus vectored by Brevipalpus phoenicis (Acari: Tenuipalpidae) on citrus in Brazil. Exp. Appl. Acarol. 30:161-179.

Roy, A., León, M. G., Stone, A. L., Hartung, J. S., and Brlansky, R. H. 2014. First report of citrus leprosis virus nuclear type in sweet orange in Colombia. Plan Dis. 98:1162.

Sanchez-Navarro, J. A., Zwart, M. P., and Elena, S. F. 2013. Effects of the number of genome segments on primary and systemic infections with a multipartite plant RNA virus. J. Virol. 87:10805-10815.

Sicard, A., Yvon, M., Timchenko, T., Gronenborn, B., Michalakis, Y., Gutierrez, S., and Blanc, S. 2013. Gene copy number is differentially regulated in a multipartite virus. Nat. Commun. 4:2248.

Sonnenberg, R., Wolte, A. W., and Tautz, D. 2007. An evaluation of LSU rDNA D1-D2 sequences for their use in species identification. Front. Zool. 4:6.

Stewart, L. R. 2016. Sequence diversity of wheat mosaic virus isolates. Virus Res. 213:299-303.

Tatineni, S., McMechan, A. J., Wosula, E. N., Wegulo, S. N., Graybosch, R. A., French, R., and Hein, G. L. 2014. An eriophyid mite-transmitted plant virus contains eight genomic RNA segments with unusual heterogeneity in the nucleocapsid protein. J. Virol. 88:11834-11845.

Thompson, J. D., Higgins, D. G., and Gibson, T. J. 1994. CLUSTAL W: Improving the sensitivity of progressive multiple sequence alignment through sequence weighting, position-specific gap penalties and weight matrix choice. Nucleic Acids Res. 22:4673-4680.

Zerbino, D. R., and Birney, E. 2008. Velvet: Algorithms for de novo short read assembly using de Bruijn graphs. Genome Res. 18:821-829.

Zheng, Y., Navarro, B., Wang, G., Wang, Y., Yang, Z., Xu, W., Zhu, C., Wang, L., Di Serio, F., and Hong, N. 2017. Actinidia chlorotic ringspot-associated virus: A novel emaravirus infecting kiwifruit plants. Mol. Plant Pathol. 18:569-581. 